\title{
A Platform Modelling with 3D-Digital Transmission Lines Engineering Design
}

\author{
Guanxing Zhang ${ }^{1, a}$, Rusong Wang ${ }^{2, b}$ \\ ${ }^{1}$ Shanghai Electric Power Japan Co.,LTD, 32F Marunouchi Bldg, 2-4-1 Marunouchi, chiyoda-ku, \\ Tokyo, Japan \\ ${ }^{2}$ Central Southern China Electric Power Design Institute of China Power Engineering Consulting \\ Group Corporation, China \\ a zhanggx@shanghaipower.co.jp, bhmsthgccsepdi@163.com
}

Keywords: Transmission Line; Digital Designing; GIS

\begin{abstract}
This paper introduces a system which is designed for the transmission design business which make it possible to achieve electrical design, structural design, technical and economic analysis, environmental design and resource sharing. This platform functions in various stages including the informatization of geographic information, path selection optimization, design results statistical query, economic indicators, and digital transfer, which greatly improves the level of consulting design services.
\end{abstract}

\section{Introduction}

With the development of science and technology, computer technology has become an indispensable tool for all works of life. In terms of design industry, the application of computer in engineering design and management has gradually become one of the symbols to measure the level of engineering design [1] .

At present, two-dimensional computer aided design and auxiliary drawing technology has been enjoying a great popularity. As a new design means, 3D digital aided design is also in the stage of vigorous development in the field of power plant, substation and other design. Its abilities of intuitive, convenient and collaborative to work have given rise to more and more attention [2] .

At this stage, some of the design companies have started trying to use three-dimensional digital design in different fields, including the transmission line tower design. However, this method has not yet been used in the true sense of the three-dimensional digital design both at home and abroad. Therefore, it is very necessary to to introduce digital three-dimensional design concept in the field of transmission line design and build a unified design platform for three-dimensional digital lines to make the design more intuitive, more convenient and improve the accuracy and efficiency. [3]- [5]

The platform has established a system of integrated design and management, which achieves collaborative work and resource sharing in three-dimensional digital transmission design. The platform provides the life cycle planning, design, analysis statistics, program optimization and other related functions of a transmission line. Covering the project planning line selection, feasibility study, bidding, preliminary design, construction design and other stages, It can complete the function in various stages including the informatization of geographic information, path selection optimization, design results statistical query, economic indicators and digital transfer, which greatly improves the level of consulting design services. 


\section{Function and Structure of 3D Digital Platform for Transmission Line}

\subsection{Transmission line three-dimensional digital platform function}

The three-dimensional digital design platform of transmission line is based on large-scale database and data high-precision image, DEM and basic geography knowledge. Relied on the three-dimensional refinement model, using aerial survey technology, 3D visualization technology, virtual reality technology and information integration technology, it combines with geographic information and engineering information, integrated transmission line corridor terrain, geomorphological information and construction process data through the data-driven model: to service for the exploration and transmission design through the construction of a real three-dimensional field environment.

The system provides complete functions of data management for the surveying business, including 4D data management, field data acquisition and mapping, data management and distribution of three-dimensional terrain, engineering parameter maintenance, survey data management and flat cross-section, house distribution map output and other functions. It can achieve the engineering data input, editing, update, delete, query, statistics, browsing and output functions by interfacing with other data management system, professional design and other software.

The System is designed for the transmission design business. It realizes electrical design, structural design, technical and economic analysis, environmental design and other functions. It also provides various professional software interface to support the follow-up business module expansion.

\subsection{The hierarchical structure of three-dimensional digital transmission platform}

Transmission line digital design system uses three-tier C/S architecture. The bottom layer provides the data access and data services, the middle layer provides business and view operations and the top layer is a presentation layer for the user to provide application.

\subsection{Database and Data Flow of Three-dimensional Digital Platform for Transmission Lines}

Data is the core of the system running. It runs through the whole process of digital systems. In the construction of the digital system, all data are collected to construct a unified transmission line database, which is consisted by engineering information base, basic geographical library, special information database, three-dimensional model library, business database, and document library respectively. Each sub-library has a clear border and clear division of labor, obeying the principles of software engineering, surveying and mapping industry and the relevant standards of power industry. The data set of the logical data model design meets the 3NF standard paradigm requirements, and is designed with CASE tools to achieve ER (physical relationship). 


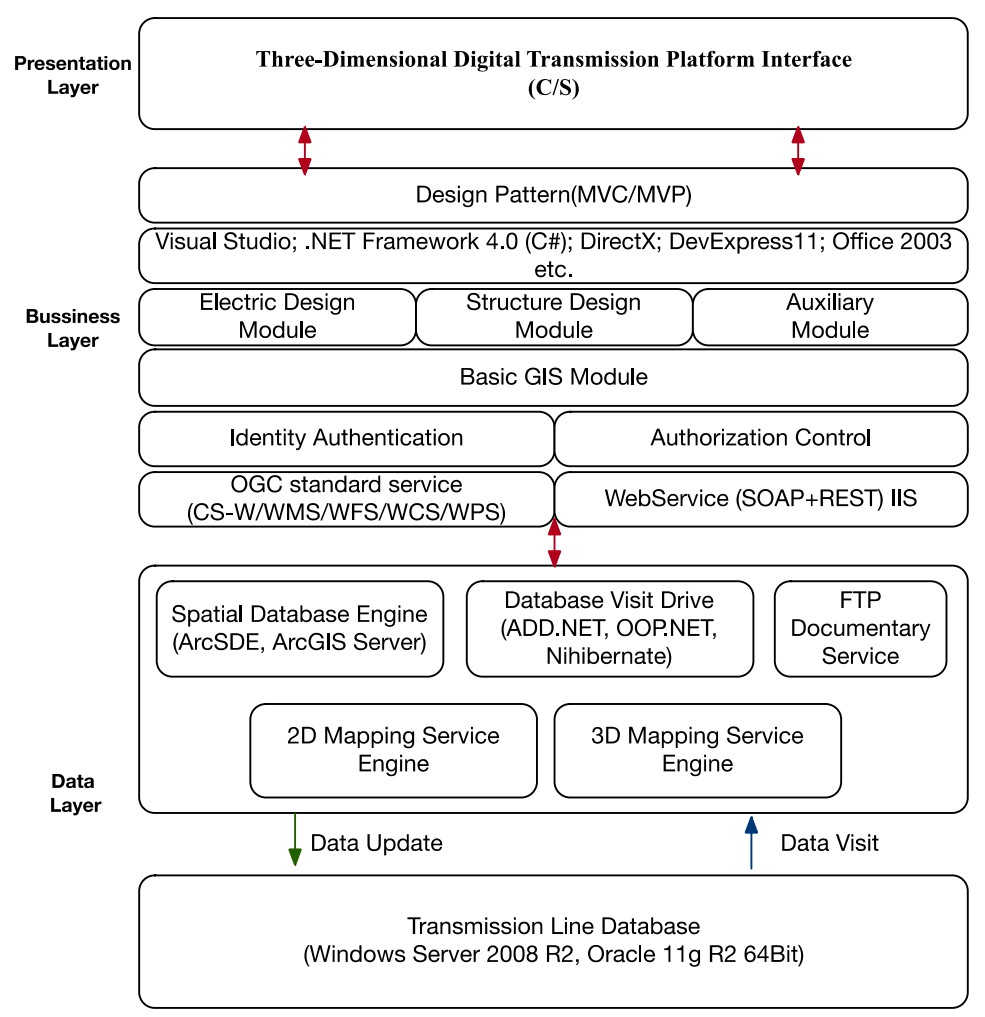

Figure 1 Three-Tier C/S Architecture

\subsection{Platform Modelling of Three-dimensional Digital Transmission Lines}

\subsubsection{Tower Model Establishment}

The system establishes the tower model to meet the accuracy requirements of different design stages by importing the tower model in the existing database, parameterizing the tower model, importing the TTA or Daoheng data file and establishing the refinement model.

\subsubsection{Establishment of the Basic Model}

The basic model is established by using the three-dimensional small scene modelling tool provided by the system. It can describe the foundation of the large plate foundation, the ladder foundation, the bolt foundation, the excavation foundation, the inclined foundation, the rock foundation and the pile foundation quantitatively. Based on the content of the parametric description of the three-dimensional model and adjustment the model style and size of parameters of real-time, it can build a basic three-dimensional model which can quick response to the needs of users. In addition to the simulation and material statistics of three-dimensional, the model can also be used to check whether the base heightening is sufficient to meet the requirements or not.

\subsubsection{The Establishment of Three- Dimensional Fitting, Insulator Set Model}

Embedded in the major manufacturers of insulators and fitting model library, a real simulation of the shape and size of various types of insulators, and fitting, based on the fine parts of the insulator set assembly. 


\section{The Innovation Application of Three - Dimensional Transmission Digital Line Platform}

\subsection{The Design of Three-Dimensional Collaborative}

The platform provides designers with a shared platform that allows all designers to operate on the same platform. It provides a real-time online convergence of different design professionals with various design results from each designers, so that different design professionals and can contact with each other and exchange ideas smoothly. Finally it will maintain the design work with the coherence ultimately to achieve a fine layout design, to avoid design errors and design omissions and other issues.

\subsection{Line Selection of Three-Dimensional Integration}

Using the high definition satellite imagery with the digital terrain model, the digital corridor can be expressed and simulated digitally by $3 \mathrm{D}$ real-world simulation technology. It takes all professional divisions of the ice area, wind area, geological conditions, seismic intensity and other basic data into the professional collaborative work platform, to achieve the route path of the multi-program line selection and technical and economic comparison by combining with the corridor information database. Based on the line path optimization options, it can realize the two-dimensional and three-dimensional positioning linkage design. The three-dimensional digital terrain model can be used to determine the terrain and geomorphology of the tower, and provided a technical reference for the site location of the tower, in order to improve the design efficiency.

\subsection{Three-Dimensional Calibration of Electric Clearance}

The three-dimensional tower model and the three-dimensional digital terrain model are used for realizing the automatic check of the electric clearance in the static state and the wind swing state, which solves the verification problems of the clearances of wire jumper to tower structure, conductor sag to tower structure, wire to wind control point, crossing span and etc.

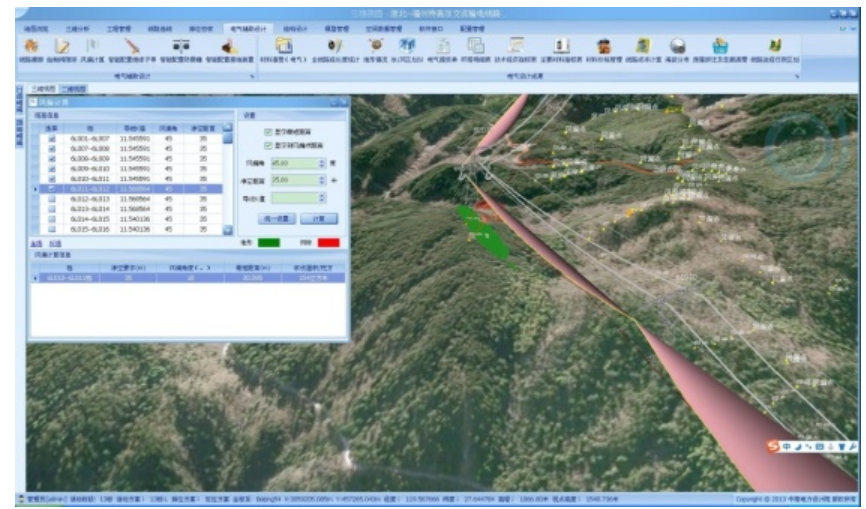

Figure 2 Clearance Checking in Tower 


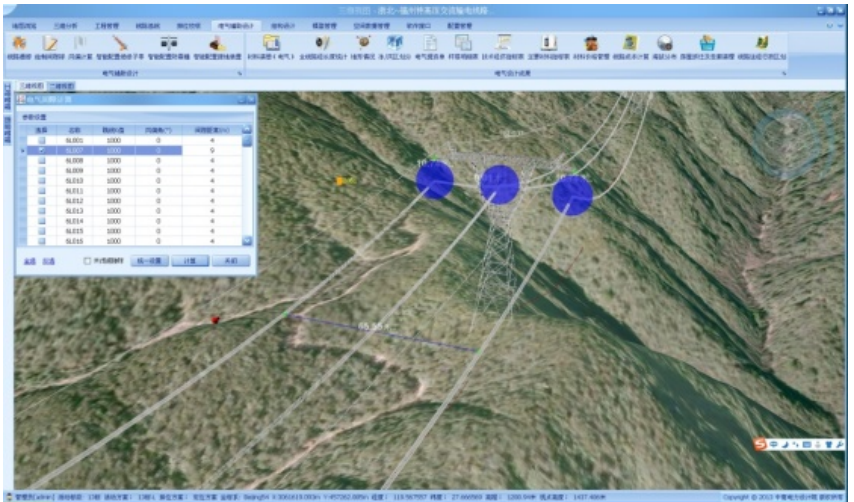

Figure 3 Clearance Checking in Span

\subsection{The Basic Configuration of Three- Dimensional}

According to the results of the electrical tower arrangement and the DEM, geology and hydrological data in the database, the basic configuration can be carried out in batches and quickly, according to the configuration result, the $3 \mathrm{D}$ simulation scene can be generated, so that the designers can view the configuration results intuitively.

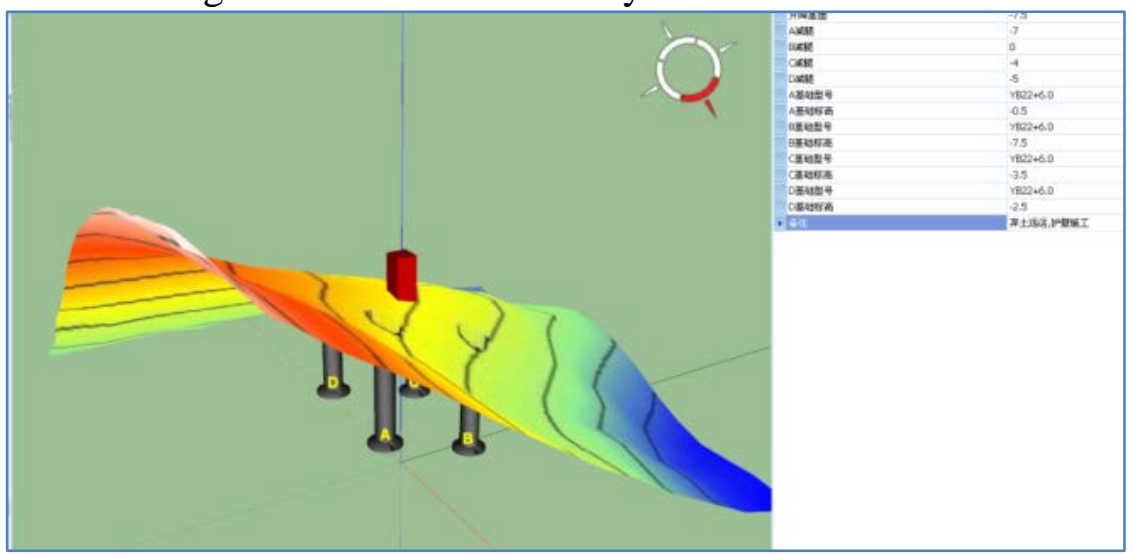

Figure 4 3D foundation configuration

\subsection{Assemble Design of Three-Dimensional Insulator}

Through the parameterized gold parts model, it is judged whether the connection point of the insulator string and the direction of the fittings is adjusted automatically, so as to avoid the connection error, guide the assemble process and improve the assemble efficiency.

It can provide a full range of automatic detection including the fittings joint, size and load damage calculation and so on to ensure the validity of the connection of the insulator string assemble.

After the completion of the design, the three-dimensional insulator string model can statistics the amount of material and complete the three-dimensional-based design work, and it can also be used to associate with the three-dimensional tower model to carry out the work of the tower clearance check.

\subsection{Virtual Reality and Digital Simulation}

Through the virtual reality technology, with high-precision image and elevation data, a real three-dimensional topography of project corridor can be simulated in the digital earth. It can assist 
designers to manage the distribution of engineering terrain more directly and vividly. Through the construction of image pyramid, LOD layer can achieve large scene data volume data browsing so as to improve the efficiency of design work.

Combined with data of three-dimensional topographic, it achieves three-dimensional mapping along the project and three-dimensional line selection to ensure that the pile is always located in the the best point. It can eliminate the problem brought by the large gap between the piles, and watch-out the path of the flat cross at any time in order to improve the accuracy of design.

Combined with high-precision tower, insulator fitting and other three-dimensional model data, it can achieve three-dimensional tower arrangement. In the process of tower structure adjusting, in real time it can finish the check the distances of tower top to land, cross span, $\mathrm{Kv}$ value and tower load. It can achieve a real-time simulation of the tower, the wire sag situation in tower adjustment process, information of the distance of early warning wire to the land, in order to eliminate the problem of cross-collision and other issues of the design. Based on the design results of the alignment check, the three-dimensional arrangement of the tower, the anti-vibration hammer and the grounding device are carried out quickly. It can reflect the engineering design data and shorten the design cycle by the main design parameters, the design data and the automatic summary of the material data.

\section{Digital Handover and Life Cycle Management}

\subsection{Digital Transmission of Transfer Work}

Taking data flow and information flow as its main line to a unified standard and norms for the protection of digital way, through the entire life cycle of the grid project, the design phase of the digital results are applied to the various stages of construction for the grid construction. It provides a unique and authoritative data source in the whole process of management.

The transfer of data is based on the choice of authorized designers, which contains the selection of the relevant content of the project from the database as a transfer of information, according to the owners of the unitary transfer format of the provisions of the unified management of the various types of data summary output. The integrity of the data is handed over to the owner of the digital handover platform precisely.

\subsection{Life Cycle Management of Digital Transmission Lines}

Transmission line digital design system support the extension, so that the project can be established through planning, feasibility study, design, procurement, processing, construction, operation and maintenance. Forward to the bidding, planning, feasibility study stage to provide support and for backward extension of the construction, completion, operation, maintenance support, and thus throughout the transmission line whole construction life cycle, and provide technical support and data support for the whole construction life cycle management of three-dimensional information.

\section{Engineering Application Example of 3D Digital Platform}

\subsection{Practical Application of 3D Digital Platform}

In the process of designing Ximeng-Shandong $1000 \mathrm{kV}$ UHV transmission line project preliminary and construction design, it work with the use of digital design platform.

In August 2014, preliminary design work started. Built a three-dimensional GIS model to realize 
the local scenic area, silver poly metal detailed investigation area, a county-level nature reserve, a reservoir nature reserve, industrial park, molybdenum mine, a large enterprise iron ore survey area, as the entry of geographic information.

In September 2014, the three-dimensional line platform selection work started. Considering the geographic information in the platform and combined with the factors such as topography, topography and altitude, the route path scheme is optimized, and the path length is reduced by about $2 \mathrm{~km}$.

In October 2014, on-site positioning work started. Combined with three-dimensional topographic data, the three-dimensional mapping and three-dimensional line selection along the project was achieved. To ensure that the pile is always located in the the best point local topography, the removal the tower that can not be a large span of the situation rapidly: keeping an eye on the choice of path flat cross at any time to improve the accuracy and efficiency of design.

In December 2014, on-site positioning work was completed.

In January 2015 - July 2015 the electrical cross-section map, tower table, the basis of construction plans, spacing bar and anti-vibration hammer installation drawings, insulator construction drawings and other volumes were published.

In July 2015 - March 2016, the digital platform was used to assist the construction unit for basic construction, tower assembly, insulator installation.

In July 2016, the project completed and was put into operation.

In September 2016, digital handover work started.

\subsection{Technical and Economic Indicators and Social Economic Benefits}

In the process of preliminary design of transmission project and construction, a three dimensional GIS model was created and a three - dimensional digital design was made. The number of towers is reduced from $2.2 / \mathrm{km}$ to $1.98 / \mathrm{km}$ in the feasibility study stage: it had reduced the total cost of the project effectively and improved the efficiency and accuracy of the basic design which saved about $130 \mathrm{~m}^{3} / \mathrm{km}$ for the concrete indicators of the project while the amount of earth and stone was saved about $800 \mathrm{~m}^{3} / \mathrm{km}$. Combined with other changes in the amount of engineering, the ontology investment reduced about 0.6 million CNY per km.

In addition, due to the characteristics of quick and accurate of the three-dimensional digital line selection, it optimized the line corridor effectively, avoiding the housing intensive area, and the house demolition quantity had been reduced about $150 \mathrm{~m}^{2} / \mathrm{km}$. Compared with the situation that without the use of three Digital platform before, the miscellaneous expenses had been reduced about 0.3 million CNY per $\mathrm{km}$. Based on the factors mentioned above, the static investment in the construction phase was 9.06 million CNY per km, which was about 0.9 million CNY per km less than the static investment 9.962 million CNY per km in the feasibility stage.

\section{Conclusion}

1). Achieve the professional co-operation on the platform, protect the design quality, and also shorten the design hours of investment. It is conducive to the optimization of the design process, authority of the design norms and improve the quality of finished products and the production efficiency.

2). Achieve the design of three-dimensional digital. In the design phase of the line terrain, features, towers, wires and other line information for digital processing, the intuitive digital model is established to make establishment of digital handover and to facilitate the construction unit for the construction schedule management by using the digital handover. Easy for the constructors to manipulate the operation and maintenance, and finally achieve the project life cycle management. 
3). With the development of 3D digital design and handover, the concept of project life cycle construction management is extended to the whole project construction, which has produced good technical and economic indicators. It has positive benefits of social demonstration, and improves the consulting design service Level greatly.

\section{Reference}

[1] YUAN Zhao-xiang, HAN Wen-jun(State Power Economic Research Institute,Beijing 100052, China); Further Application of the Helava Technology in the Whole-Process of Power Grid Construction[J]; Energy Technology and Economics; 2010-12

[2] MEI Nian, CHEN Dong, DU Xiaolei, YANG Yuan, WANG Zan, CHENG Wei (State Power Economic Research Institute, Beijing 100052, China); Three-Dimensional Digitalization Transfer of Qinghai-Tibet $\pm 400 \mathrm{kV}$ DC Interconnection Project[J]; Electric Power Construction; 2012-05

[3] Gao Yi1,Yuan Jing-zhong1,Wan Ming-zhong2,Jiang Rong-an2(1.North China Grid Company Limited,Beijing 100053,China;2.Beijing Northstar Digital Remote Sensing Technigue Co.Ltd.,Beijing 100011,China);Application of 3D Digital Simulative Technology Platform to Assisting Construction of Grid Project[J];North China Electric Power;2009-03

[4] HUANG Ping(State Power Economic Research Institute,Beijing 100761,China);Prospects of Application of GIS in Power Grid Planning[J];Electric Power Technologic Economics;2009-04

[5] DING Yongfu1,LIU Hao1,WAN Mingzhong2,XIAO Shaohui2(1.Construction Department of State Grid Corporation of China,Beijing 100031,China;2.Beijing North-Star Digital Remote Sensing Technology Co.Ltd.,Beijing 100120,China);Three-Dimensional Digitalization Transfer of Ningdong-Shandong $\pm 660 \mathrm{kV}$ DC Power Transmission Pilot Project[J];Electric Power Construction;2011-02 